\title{
ICLO DE VIDA Y ASPECTOS MOTIVACIONALES DE UN GRUPO DE INVESTIGACIÓN
}

Life cycle and motivational aspects of a research group*

(ID) Madelyn Matabanchoy Salazar*

(ID) Oriana Teresa Ruiz Bravo ${ }^{* *+}$

(ID) Fredy Villalobos Galvis ${ }^{*+\alpha+}$

\section{(C) $10(0)$}

* Surge del proceso de autoevaluación y reformulación de la plataforma estratégica del Grupo de Investigación Psicología y Salud de la Universidad de Nariño

** Universidad de Nariño, Grupo de investigación Psicología y Salud, Pasto-Colombia, E:mail: madlin.mta@gmail.com

*** Universidad de Nariño, Grupo de investigación Psicología y Salud, Pasto-Colombia, E:mail: orianaruizbravo@hotmail.com

**** Universidad de Nariño, Grupo de investigación Psicología y Salud, Pasto-Colombia, E:mail: fhvillalobos@gmail.com

Fecha de recepción: 25 de abril del 2018

Fecha de aceptación: 31 de mayo del 2018

\section{Cómo referenciar / How to cite}

Matabanchoy-Salazar, M., Ruiz-Bravo, O. T., y Villalobos-Galvis, F. (2018). trilogía Ciencia Tecnología Sociedad, 10(19), 149-164. 
Resumen: tradicionalmente, un Grupo de Investigación (GI) es definido como una unidad funcional que se enfoca primordialmente en procesos de investigación cientifica y de productividad académica, no obstante, las definiciones no suelen tomar en cuenta las dinámicas grupales e individuales que se dan a su interior a lo largo de su vida como grupo. Por esto, el presente estudio tiene como propósitos: formular una propuesta teórica sobre el ciclo de vida de un GI, identificar los aspectos motivaciones de sus integrantes y, desde lo anterior, aportar elementos que permitan la formulación de una nueva concepción de GI. Para ello, se llevó a cabo un estudio cualitativo que implicó el análisis de la información recolectada durante los años 2014 y 2015, en el marco del proceso de autoevaluación del Grupo Psicología y Salud, en el cual participaron docentes y profesionales investigadores. En este proceso se aplicaron técnicas de recolección de información tales como: encuestas con preguntas abiertas, grupos focales y una entrevista cualitativa; estas técnicas permitieron la obtención de datos significativos que posteriormente fueron analizados y categorizados a partir de elementos de la teoría fundamentada. Se identificaron tres tipos de motivaciones de los integrantes al momento de su vinculación al grupo, se planteó un modelo sobre el ciclo de vida de un GI, y por último, se propuso una definición integral que permita reconocer a los GI como espacios humanos y sociales que trascienden a la mera productividad científica. Estos resultados pueden brindar herramientas a las instituciones y a los mismos GI para que formulen políticas y estrategias integrales, centradas en el talento humano en investigación y destinadas a la gestión de conocimiento.

Palabras clave: grupo, investigadores, investigación, motivación, ciclo de vida.

Abstract: Research groups have been traditionally described as functional units that primarily focus on scientific research processes and academic production. However, these definitions do not generally consider the team and individual dynamics that take place during their life cycle. This study aims to propose a theory of the life cycle of research groups, identify the motivational aspects of its members, and (based on that) provide elements to create a new definition. For this purpose, a qualitative study analyzed information collected in 2014 and 2015 from a self-evaluation process of the group Psicología y Salud in which teachers and research professionals participated. The process applied information collection techniques such as surveys with open questions, focus groups and a qualitative interview. Said strategies enabled to obtain significant data that were later analyzed and categorized based on elements of the Grounded Theory. Three types of motivations to join the group were identified and a life cycle model was proposed. Finally, we suggest a comprehensive definition that recognizes research groups as human and social spaces that transcend scientific productivity. These results can provide institutions and said groups with tools to develop comprehensive policies and strategies focused on human talent in research and aimed at knowledge management.

Keywords: Group dynamics, research, motivation, research policy. 


\section{INTRODUCCIÓN}

En Colombia, el Departamento Administrativo de Ciencia, Tecnología e Innovación -Colciencias (2017a) define a un Grupo de Investigación (GI) como un «conjunto de personas queinteractúan para investigar y generar productos de conocimiento en uno o varios temas, de acuerdo con un plan de trabajo de corto, mediano o largo plazo (tendiente a la solución de un problema)» (p.23). Esta definición orienta su intención al fin último del grupo, es decir, a la generación de conocimiento científico, sin embargo no enfatiza en el hecho de que es un «conjunto de personas que interactúan», dejando de lado el elemento social y humano que implica una organización como el grupo. En este sentido, Kuhn (2006) afirma que los grupos académicos comparten conocimientos especializados, metodologías e instrumentos propios de un campo. Parecería que los únicos elementos que vinculan a los integrantes de un GI fueran aquellos propios de un método o de un conocimiento científico específico. Sin embargo, pensar que los investigadores son personas, seres humanos, con características que los hacen únicos y particulares como cualquier otra persona, genera la necesidad de comprender a los GI desde una dimensión interna, social y organizacional que permita, en primer lugar, concebir a este tipo de grupos como un todo dinámico, integrado por dos o más personas, quienes se relacionan, influyen y son influidas a su interior (Shaw, Robbin, \& Belser, 1981); y en segundo lugar, entender que un GI no es solo un conjunto de personas que realizan investigación, sino que ellas cuentan con personalidades, habilidades, capacidades $\mathrm{y}$ disposiciones afectivas y emocionales, que le dan variabilidad, sentido y dinámica al grupo, permitiendo también una cultura de convivencia que además de lo social, influye igualmente en su proceso investigativo (Londoño, 2005).

Concebir al grupo como una unidad dinámica, significa que tanto este como sus integrantes están en constante cambio y en ese sentido se encuentran propuestas teóricas que sugieren que los grupos atraviesan por una serie de momentos durante su existencia. A continuación se refieren estudios en los cuales se presentan modelos del proceso de formación de grupos.

El modelo formulado por Tuckman y Jensen $(1965,1977)$ y retomado por Altopiedi, Hernández y López (2015), se convirtió en un referente inicial para comprender a los grupos en general, como entidades dinámicas sujetas a ciclos evolutivos; en ese sentido, los autores plantearon que tanto los grupos como los equipos se desarrollan a través de cinco etapas: formación, confrontación, regulación, desempeño y renovación. Si bien este modelo es útil para comprender las dinámicas de diferentes grupos, se hace necesaria la identificación de modelos evolutivos propios y específicos que permitan entender la dinámica de los GI.

Acorde a lo anterior, Hamui (2011) describe la experiencia vivida por un equipo de investigación en el desarrollo de un proyecto, diferenciando tres momentos durante el proceso de trabajo: el primero, denominado conformación, correspondió a la realización de un proyecto de investigación, durante el cual se alcanzaron aprendizajes y se establecieron compromisos para lograr los objetivos; en el segundo momento, la transición a la consolidación, algunos valores se fueron consolidando y re-significando, destacándose el compromiso de los investigadores con los procesos desarrollados; y para la etapa final, de consolidación, los integrantes generaron los resultados del proyecto, presentándose un ambiente de tensión, reconocimiento, competencia y crítica. Este antecedente es pertinente, pues muestra los procesos acaecidos en la dinámica social de las personas que conforman el equipo. Sin embargo, a pesar de que muestra la dinámica grupal a lo largo del proceso de desarrollo de una investigación, no alcanza a formular una propuesta que permita entender la evolución de un GI más allá de la realización de un proyecto de investigación, por lo que se requiere una mirada más amplia que responda a un GI con una perspectiva de funcionamiento a mediano y largo plazo. 
Al respecto, Rey-Rocha, Martín y Sebastián (2008) amplían y generalizan un modelo específico para los GI, que integra ciclos de vida, delimitados por las etapas de generación, crecimiento y segregación. Según el modelo, el origen de los GI se da a partir de la asociación de un investigador senior con varios investigadores; luego con la fase de crecimiento, se presenta la capacidad de vinculación de nuevos integrantes, quienes aportan una diversidad al grupo; y por último, en la segregación o gemación, los integrantes deciden salir del GI debido a diferentes razones como tener mayor independencia, conflictos con el grupo o nuevas oportunidades de desarrollo profesional. Como se puede observar, este modelo ofrece una mirada un poco más longitudinal de la vida de un GI; sin embargo, está formulado con la idea de que el grupo termina con las etapas de segregación o gemación, mas no plantea qué sucede si el GI busca perdurar en el tiempo, a pesar de estos fenómenos de crisis y deserción.

Las propuestas anteriormente mencionadas evidencian una falta de consenso sobre cómo se desarrolla un GI, es decir, sobre cuáles son las etapas por las que pasa durante su vida, lo cual puede deberse a que cada grupo tiene un estilo particular que refleja su dinámica y sus respectivos procesos grupales de liderazgo, trabajo en equipo, comunicación entre sus integrantes, compromiso y clima de trabajo (Omar \& Ahmad, 2014). Otra explicación es que existe una carencia significativa de investigaciones que se centren en cómo funcionan y se desenvuelven los GI, lo que impide profundizar y ampliar las propuestas anteriores, de cara a una mayor comprensión y a abordajes pertinentes.

Por otra parte, y en complemento con lo que anteriormente se abordó, se puede afirmar que las dinámicas grupales, los procesos sociales de liderazgo, la colaboración mutua, la disposición de espacios para compartir y crear conocimiento, y los aspectos motivaciones de los investigadores, determinan el adecuado desempeño y

Vol. 10 • Núm. 19 / jul-dic / 2018 productividad del GI, por ello es un deber de las instituciones reconocer dichos factores para convertirlos en fortalezas dentro de los grupos (Gómez \& Monserrat, 2015).

Al respecto, Gómez y Montserrat (2015) manifiestan que la motivación en los integrantes del GI, además de estar vinculada a la carga laboral, está mediada por el valor que se le otorga al aprendizaje y la experiencia adquirida en la generación de nuevos conocimientos. Igualmente, González (1979) afirma que al ser los recursos económicos para la investigación científica tan limitados en ciertos campos, no se puede pensar únicamente en una motivación centrada en una remuneración económica, sino que la comunidad científica debe enfocarse en las razones y motivos que movilizan el accionar de los investigadores, y de esa forma, plantear acciones en pro de su permanencia dentro de los GI.

En ese sentido, y teniendo en cuenta que la investigación dentro de las instituciones depende en gran medida de sus GI, se plantea la necesidad de generar políticas en ciencia, tecnología e innovación, que no solo se centren en aspectos netamente productivos de los GI o en los recursos económicos y materiales que puedan necesitar, sino también incluir dentro de estas la gestión del talento humano que integra a dichos grupos (Lastra, 2017; Ceballos, Garza \& Cantu, 2018), comprendiendo que los GI no se componen de máquinas productoras de conocimiento, sino de personas que sienten, se frustran, aprenden y comparten su vida con colegas, con características personales que modulan su funcionamiento y su productividad, e influyen en el interés que tengan de formar parte de un grupo y de permanecer en él (Isohanni et al., 2002).

En coherencia con lo expuesto, para comprender la dinámica de funcionamiento de un GI, se requiere denotar que tanto las variaciones sociales como individuales se relacionan entre sí y generan un funcionamiento único de cada grupo. Es por 
ello, que el presente artículo tiene tres propósitos fundamentales: formular una propuesta teórica sobre el ciclo de vida de un GI; identificar los aspectos motivaciones de sus integrantes $\mathrm{y}$, desde lo anterior, aportar a la formulación de una nueva concepción de GI; todo esto con el fin de brindar herraminentas a las instituciones para que realicen un adecuado ejercicio de reflexión y toma de decisiones de cara a la construcción de políticas que apoyen la actividad científica de los GI.

\section{MÉTODOLOGÍA}

Se llevó a cabo un estudio de tipo cualitativo, con elementos de la metodología de análisis de Teoría Fundamentada (Strauss \& Corbin, 2002; Gómez y Villalobos, 2014) y que tuvo en cuenta la experiencia vivida por los investigadores de un GI. Para ello se tomó como insumo la información recolectada durante los años 2014 y 2015 en el marco del proceso de autoevaluación y diagnóstico de este grupo, el cual estaba orientado a definir su nueva plataforma estratégica. Se contó con información relacionada con aspectos sociales e individuales que se desarrollaban al interior del GI, por medio de técnicas cualitativas que permitieron un acercamiento a las percepciones y representaciones de los integrantes del GI con respecto a su vinculación y experiencia dentro del mismo, logrando una comprensión del fenómeno $\mathrm{y}$, por ende, una reconstrucción de su realidad (Hernández, Fernández \& Baptista, 2014).

\section{Participantes}

La unidad de análisis del presente estudio correspondió al GI Psicología y Salud, adscrito al Departamento de Psicología de la Universidad de Nariño. Su creación se remonta al año de 1999, a partir del cual ha presentado un adecuado nivel de desempeño y continuidad en sus labores científicas, formativas y de proyección social (Colciencias, 2017b, p.140). El GI actualmente se encuentra conformado por 28 integrantes con diferente nivel de cualificación profesional, como estudiantes, profesionales o docentes. La unidad de trabajo la integraron 18 investigadores, 12 mujeres y 6 hombres, de los cuales 9 eran docentes y 9 eran profesionales, cuyos niveles de formación académica variaron de la siguiente manera: 7 con estudios de pregrado, 2 especialistas, 3 maestrantes, 4 magísteres y 2 estudiantes de doctorado. Se estableció como criterios de inclusión que las personas fueran integrantes activos y que tengan más de un año de pertenencia al grupo.

\section{Técnicas e instrumentos}

Para la recolección de información se elaboró una encuesta estructurada con preguntas abiertas, en la que se indagó aspectos como la fecha de ingreso de los integrantes, los motivos personales frente a su vinculación, las funciones desempeñadas, el proceso histórico del grupo (inicios, hitos y aspectos de cambio o recomendaciones), la plataforma estratégica, la productividad académica y los factores de reconocimiento institucional hacia los productos.

Por otra parte, se realizó la contrucción de la matriz DOFA (Debilidades, Oportunidades, Fortalezas y Amenazas), con el propósito de identificar el perfil operacional del GI, con miras a la formulación de su nueva plataforma estratégica. Para este proceso se hizo uso de las técnicas cualitativas de grupo focal (Hernández, Fernandez y Baptista, 2014) y roleplaying (Crano \& Brewer, 1973).

Por último, se desarrolló una entrevista cualitativa (Hernández, Fernández y Baptista, 2014) al director del GI, con el objetivo de profundizar información sobre la vida e historia del GI Psicología y Salud, en lo relacionado con su devenir histórico, hitos en su desarrollo y forma de trabajo de los integrantes.

\section{Procedimiento}

El proceso de recolección de la información se llevó a cabo a lo largo de tres etapas: la primera de forma individual, en la cual se aplicó la encuesta 
154 estructurada en formato online oen formato de papel y lápiz a cada uno de los participantes (de acuerdo con su disponibilidad de trabajo), presentando el respectivo consentimiento informado para el manejo de la información con fines académicos. En la segunda etapa se aplicó la estrategia DOFA (Ponce, 2007) que permitió la construcción del perfil de capacidades internas (fortalezas y debilidades), y la identificación de amenazas y oportunidades para el GI, la cual se realizó en sesiones diferentes en las que se construyó y validó grupalmente las categorías que se identificaron. Finalmente, en la tercera etapa se realizó la entrevista cualitativa con el director del GI.

\section{Analisis de datos}

El análisis de datos de la información recolectada se hizo desde la Teoría Fundamentada, en la cual se realizó una codificación abierta, una codificación axial y una codificación selectiva (Strauss \& Corbin, 2002). Estos tipos de codificación más que entenderse como procedimientos secuencialmente separados y diferentes, fueron concebidos como un manejo de datos con distintos grados de abstracción (Flick, 2004). Para dicha codificación, se hizo uso del método de comparaciones constantes, el cual permite reconocer las relaciones entre datos y construcciones conceptuales de los mismos, e identificar las propiedades y dimensiones de las categorías que van surgiendo del análisis realizado (Strauss \& Corbin, 2002; Raymond, 2005).

\section{RESULTADOS Y DISCUSIÓN}

Desde la recolección y el análisis de la información se construyeron tres categorías inductivas. La primera correspondió a un enfoque individual, centrado en los motivos que tienen los investigadores para integrar un GI; en la segunda se abarcó su ciclo de vida, y en la última categoría se articularon las anteriores en una definición integral de lo que se entendería como GI (Ver Tabla 1).

Tabla 1. Matriz de categorías inductivas

\begin{tabular}{ll}
\hline Categoría analítica & Subcategorías descriptivas \\
\hline 1. Motivos para la & 1.1 Motivos individuales \\
vinculación a un GI & 1.2. Motivos sociales \\
& 1.3 Motivos institucionales \\
\hline & 2.1. Definición y Formulación \\
& 2.2. Consolidación \\
2.3. Reconocimiento \\
2. Ciclo de vida del GI & 2.5. Transformacion \\
& 2.5.a. Transición \\
& 2.5.b. Gemación \\
& 2.5.c. Fin del grupo
\end{tabular}

3. Aportes a la Definición

de GI

\section{Motivos para la vinculación a un GI}

Los motivos son aspectos por los cuales las personas dan razón de sus comportamientos en función de alcanzar un objetivo o meta precisa, convirtiéndolos en una disposición para realizar determinadas acciones (Nieto \& Gómez, 2015).
En este caso, los motivos responden al interés de vincularse a un GI, por lo tanto, la motivación personal de los investigadores es un factor que influye en su productividad académica, de tal forma que se debe procurar que haya una coherencia entre los motivos de los integrantes y los objetivos que establezca el GI (González, 1979). Los motivos 
encontrados se clasificaron en individuales, sociales e institucionales.

\section{Motivos individuales}

Son aquellos relacionados con los beneficios propios que puede obtener una persona cuando ingresa a un GI, los cuales responden a un logro y a una satisfacción individual. Los encontrados se clasificaron en motivos de aprendizaje y motivos de desarrollo de investigación.

\section{Motivos de aprendizaje}

Este tipo de motivos se centra en el proceso de formación del investigador e integra el aprendizaje de habilidades, la adquisición de conocimientos, el fortalecimiento de competencias investigativas y el desarrollo tanto personal como profesional, lo cual se evidencia en frases como «fortalecer las competencias investigativas como integrante del grupo» (E-S1) ${ }^{1}$ y la «posibilidad de potencializar habilidades investigativas» (E-S13). Lo anterior se relaciona con lo planteado por Fuentes (2015) al manifestar que existe un tipo de investigación formativa que permite a los investigadores generar un ambiente de aprendizaje y desarrollo de capacidades investigativas al interior de los GI.

\section{Motivos de desarrollo de in vestigación}

Se relacionan con aspectos como la realización de proyectos, artículos, ponencias, la verificación de teorías, entreotras accionesquepermitan ala persona adquirir una experiencia investigativa en un campo o tema específico. Entre los motivos encontrados se destacan, «hacer parte de investigaciones dentro de la Universidad de Nariño y la región»(E-S1), «la experiencia en investigación» (E-S5) y «mi interés por la investigación» (E-S8). Además se evidenció, que la mayoría de los participantes que se vincularon al grupo cuando eran estudiantes, lo

${ }^{1}$ Los textos de las encuestas realizadas a los integrantes del grupo se codificaron como E-S\#, en el que E corresponde a encuesta, $S$ a sujeto y \# a la numeración interna asignada a los participantes. hicieron con el interés de desarrollar su trabajo de grado.

De esa forma, los motivos individuales guardan relación con los motivos de logro (Romero, 1999), los cuales implican alcanzar actividades con significado personal a través de metas, generalmente intrínsecas, que responderían al interés de aprender e investigar dentro de un GI, proceso que implica una experiencia significativa en el investigador, permitiendo que asuma un rol que lo movilice internamente y ocasione una transformación personal en él (Kyvik, 2013; Clavijo, 2014).

\section{Mdivossodiales}

Son aquellos motivos que implican una constante relación y comunicación con otra persona, y se centran en su interacción; estos motivos llevan a que los investigadores busquen relacionarse entre pares, no solo para generar conocimiento, sino también por el sentido mismo de encontrar interacción entre ellos (Martínez, 2013). Para esta categoría se proponen tres tipos de motivos: los de afiliación, los que se centran en la experiencia del otro como fuente de aprendizaje y los de construcción y validación colectiva del conocimiento.

\section{Afiliación}

Corresponden al interés de pertenecer a un grupo, ser parte de él y tener la oportunidad de entablar relaciones cercanas y amistosas con sus integrantes. Entre los discursos de los investigadores encontramos «quiero resaltar el sentido de pertenencia y como lo decía antes, el alto talento humano con que cuenta el grupo» (E-S9) y «agradecer al grupo por los aportes brindados, por permitirme hacer parte de él» (E-S12). Lo anterior se relaciona cuando McClelland (1989) describe a la motivación de afiliación como la búsqueda de estar en la presencia de otros para compartir creencias, sentimientos y experiencias. 
La experiencia del otro como fuente de aprendizaje

El segundo motivo social resalta la importancia de la experiencia investigativa de los antiguos miembros, como una oportunidad para el desarrollo de conocimientos y habilidades para el nuevo integrante. Esto implica el reconocimiento de la trayectoria investigativa, cualidades y área de conocimiento en la que trabajan los integrantes. Entre los relatos encontrados se destacan «los aprendizajes que puedo obtener gracias a las producciones de investigación de los compañeros» (E-S7), el «aprender de los investigadores que hacen parte del grupo quienes tienen experiencia en investigación en distintos campos» (E-S8) y el «aprender a investigar al lado de docentes con trayectoria en el área»(E-S16). Lo descrito tiene coherencia con el estudio realizado por González (1979) cuando expone que un interés de los investigadores es el de trabajar y relacionarse con colegas de alta experiencia y calidad investigativa.

\section{Construcción y validación colectiva de conocimiento}

Porúltimo, seencuentranlos motivosqueconsideran al GI como un espacio en donde se construye y valida el conocimiento colectivamente, haciéndose necesaria la opinión del otro, la comunicación y el trabajo en equipo. A este respecto se encontraron frases como «tener la oportunidad de compartir con investigadores, aplicar los conocimientos que como psicólogos poseemos, verificar las teorías existentes y poder hacer un contraste entre ellas y la evidencia obtenida durante el desarrollo de las investigaciones» (E-S1) y «vivir la experiencia de construcción colectiva del conocimiento»(ES5). Frente a esto, Blanco, Caballero y De la Corte (2005) manifiestan que existe una necesidad de la persona por alimentar su estructura cognitiva a través de la relación con los otros, de esa forma se valida la realidad social por medio de experiencias, opiniones y creencias de los demás convirtiendo a los GI en espacios de aprendizaje colectivo, y no como una integración administrativa en la que las personas trabajan de forma independiente (Conde \& Castañeda, 2014).

\section{Mdivosindituriondes}

Estos motivos hacen alusión al conocimiento que tienen las personas de las características de un GI y los beneficios formales que conlleva estar vinculado a este, resaltando aspectos como: la trayectoria del grupo, el apoyo formal y la proyección.

\section{Trayectoria del GI}

En este apartado se encuentran los motivos relacionados con el reconocimiento social con el que cuenta el GI Psicología y Salud. Este tipo de motivos fue expresado en su mayoria por los estudiantes que se vinculaban al grupo con miras a desarrollar sus trabajos de grado: «fortalecer mi experiencia en investigación con un grupo de trayectoria importante» (E-S5), «la posición del grupo dentro de Colciencias y su recorrido en investigación» (E-S8) y «su rigurosidad y trayectoria» (E-S18). Al respecto, Bianco y Sutz (2005) afirman que la trayectoria se expresa tanto en la producción académica del grupo, como en el reconocimiento de liderazgo en sus miembros.

\section{Apoyo formal}

Estos motivos hacen referencia al respaldo de la institución a la cual pertenece el GI, al desarrollo de los procesos investigativos de sus integrantes; por ejemplo, la formulación de proyectos de investigación, la presentación a convocatorias, la gestión de recursos, entre otros procesos que evidencien que el aval del grupo favorece al investigador. Dichas razones se complementan a su vez, con el interés institucional de cumplir los objetivos de producción académica. Entre los motivos relevantes se manifestaron: «la posibilidad de participar en convocatorias de investigación» (E-S3) y «son muchos los motivos que influyeron en mi decisión de hacer parte del grupo, sin embargo rescato como principal razón, las oportunidades académicas» (E-S12). 
Lo anterior es coherente con el estudio realizado por Rey-Rocha, Garzón y Martin (2006), quienes evidencian que el pertenecer a un GI bien establecido, proporciona a sus miembros ventajas relacionadas con mayores niveles de actividad y producción científica, además de un mayor grado de prestigio profesional en sus investigadores.

\section{Proyección}

Finalmente, encontramoslosmotivosinstitucionales que permiten trascender e impactar escenarios externos a los investigadores y al GI en sí, es decir, motivos que se centran en aportar a otros contextos, desde un sentido un poco más filantrópico. Entre los motivos destacables se encuentran «trabajar en equipo por mejorar los procesos del programa de psicología» (E-S15) y «la posibilidad de aportar desde la academia a la región» (E-S16). Esta última afirmación, encuentra relación con lo planteado por Martínez (2015) cuando refiere que la actividad investigativa no solo debe quedarse dentro del contexto universitario, sino trascender a la solución de problemas en contextos grupales, empresariales y comunitarios, lo que es coherente con la responsabilidad social que puede darse en el proceso de producción de conocimiento, y cómo los investigadores pueden incorporar los intereses de otras personas o grupos vinculados al desarrollo científico y tecnológico a través del impacto social en sus actividades investigativas (Londoño, 2005; Gaete, 2010).

\section{Ciclo de vida del grupo}

Rey-Rocha, Martín y Sebastián (2008) definen a los GI como «entidades dinámicas sujetas a ciclos evolutivos» (p. 751), en coherencia con lo anterior, para el caso del GI Psicología y Salud, se entendió al ciclo evolutivo como un proceso de constante cambio, que con el transcurrir de sus diferentes fases, le permitió al grupo un nivel diferente de complejidad (Ver Figura 1).

En el inicio de la espiral se presenta la etapa de definiciones y formulaciones, la cual corresponde a la creación del GI a partir de alianzas generadas entre investigadores quienes conformaron al grupo, «entonces nos dimos cuenta que tendríamos más que unirnos, porque éramos tres que en ese momento estábamos haciendo investigación, nadie más tenía un compromiso claro» (Director grupo, DG). A partir de lo anterior, se comienzan a establecer líneas de investigación y planes de trabajo y se orientan esfuerzos en pro de la consolidación del grupo, para lo cual se necesitó la clarificación de roles de cada investigador.

En esta fase, tienen protagonismo los primeros proyectos y acciones, puesto que permitieron a los integrantes y al grupo, adquirir experiencia y conocimiento, convirtiéndose en una etapa de aprendizaje y de auto-reconocimiento de sus capacidades, como se refleja en lo siguiente, «¡Para mí la conclusión es que se puede!, nosotros aprendimos eso: ¡se puede!, ¡se puede hacer investigación!, ¡se puede trabajar en equipo!, ¡se puede aspirar a proyectos!, para mí era como romper un poco la inercia y ver que sí nos podíamos mover como equipo» (DG).

Como segunda fase se encuentra la etapa de consolidación, la cual correspondió al despliegue de acciones para alcanzar los objetivos propuestos en la etapa anterior, a partir de diferentes modalidades de productividad, ya sea a través de la construcción, difusión o apropiación social de conocimiento. Normalmente implica la formulación y desarrollo de proyectos de investigación, la dirección de trabajos de grado de pregrado, la oferta de cursos de postgrado y la participación en eventos especializados, entre otros; lo anterior se evidencia en, «se empieza a trabajar mucho más con el tema de productividad, en el que la gente tiene mucha más efectividad a la hora de generar productos investigativos» (DG).

La siguiente etapa corresponde al reconocimiento de procesos o productos, en la cual el grupo, como fruto de la etapa anterior, comienza a ser reconocido por la comunidad académica en la que 
se encuentra inmerso, por ejemplo, «siento que fue un momento de cosechas y de tranquilidad de lo que se venía haciendo, se había consolidado y le habíamos tomado el ritmo a todo y ya teníamos como claridad frente a las cosas» (DG). Lo anterior implica que en la fase de consolidación se hayan desarrollado óptimamente las actividades propuestas, y se refleje en el reconocimiento por parte de los órganos acreditadores hacia los GI (p.e. Colciencias).

Siguiendo a la anterior, se encuentra la etapa de crisis, entendida como aquella situación que requiere de cambios relevantes o toma de decisiones estructurales para el funcionamiento adecuado e ideal del GI. Las causas de dichas crisis se condensaron en 3 situaciones: la función que tiene el GI, los factores externos que influyen en él y los factores internos que lo afectan.

Con respecto a las crisis relacionadas con la función del GI, una premisa importante a entender es que un grupo tiene objetivos específicos a alcanzar que guían su funcionamiento. Para la consecución de dichos objetivos, se pueden configurar tres situaciones de crisis: la primera sucede cuando los objetivos proyectados son simples y han sido alcanzados, «en este punto es necesario que se actualicen nuestros objetivos y líneas de investigación direccionando las acciones a seguir» (Taller DOFA-TD); la segunda situación se da cuando los objetivos formulados en un inicio fueron inalcanzables, llevando a realizar muchas acciones para cumplirlos, pero con la frustración de no lograrlos; y por último, la tercera situación de crisis corresponde a cuando los objetivos del GI no son coherentes con los objetivos e intereses individuales de los investigadores, lo que podría terminar en la insatisfacción de sus integrantes.

Con respecto a la crisis ocasionada por factores externos, se encuentran las situaciones que no dependen del grupo pero que pueden influir en su funcionamiento, por ejemplo, cuando la oferta del grupo no es coherente con las demandas del contexto, la financiación en proyectos de investigación es limitada o existe un limitado acceso a buscadores especializados y poco manejo de software para investigación, «aparecen nuevas necesidades de investigación y esto nos hace evaluar si lo que estamos haciendo y las líneas de investigación son coherentes con lo que se requiere para este momento (...) ¿Si estamos respondiendo como grupo a las necesidades que se presentan?» (TD).

Por último, se encuentran las crisis causadas por factores internos del GI, integrados por los procesos y situaciones que se presentan a su interior, por ejemplo, cuando existe un inadecuado liderazgo del grupo; el trabajo en equipo no es eficiente o es inexistente; se presentan fallas en la comunicación y divulgación de los trabajos desarrollados o se establecen normas limitantes o poco claras para el rol de cada integrante, «considero que es necesario que se planteen estrategias de comunicación para que podamos conocer qué están haciendo los otros equipos de trabajo (...) es necesario que haya mayor compromiso por parte de todos con respecto a la asistencia a las reuniones y participación en los procesos del grupo y me parece que se deben establecer criterios de ingreso y permanencia para los integrantes del grupo» (TD).

Según lo descrito anteriormente, en esta etapa se presentó un alto componente emocional y afectivo, que permitió al GI prestar atención a los procesos o aspectos que estaban fallando y debían ser mejorados. «De la crisis aprendimos esto: 1) que nada es perfecto y que es factible que muchas cosas no funcionen bien; 2) que necesitamos momentos en los cuales evaluemos y digamos que algo no funciona... Cuando uno tiene la oportunidad de ver que algo falla, ya puede asumir el reto de pensar qué hacer para mejorarlo, si no tuviéramos crisis podríamos seguir pensando que todo va a seguir igual o que debería seguir igual y si es así ¿qué interés habría por cambiar las cosas?»(DG). 
Finalmente, en la etapa de transformaciones del GI, se pueden presentar tres escenarios diferentes: Transición, Gemación o Fin del grupo. El primer escenario se da cuando el grupo concluye que requiere de transformaciones en su actuar a través de una evaluación de la crisis que lo afectó, como se manifiesta en la siguiente frase «pero lo otro que apareció es que no podíamos seguir de la misma manera, entonces se plantea la necesidad de hacer la evaluación... como el ejercicio que se hizo de autoevaluación, para mí fue como importante porque fue como empezarle a darle voz a la gente» (DG). Esta transición correspondería a ese momento de valoración y afrontamiento de las crisis que evidenciaría el esfuerzo del GI por mantenerse vivo, lo que permitió el desarrollo de nuevos procesos de definiciones y formulaciones.-

En este punto aparece la necesidad de evaluar esos factores grupales internos (p.e. comunicación, liderazgo, roles, responsabilidades, etc.), como también los aspectos individuales de cada uno de los integrantes (p.e. expectativas, intereses, necesidades, metas), llevando a que el ciclo de vida planteado, vuelva a iniciar pero a un nivel de complejidad diferente «ahora es como si yo estuviera de nuevo cuando el grupo arrancó, porque son nuevos actores, nuevos momentos, nuevos intereses, definimos nuevos objetivos, nuevas líneas y volvemos otra vez a arrancar pero ya sabemos que no estamos en el nivel de hace 10 años, estamos en otro nivel» (DG).

El segundo escenario de transformación es la gemación, que se puede dar cuando los liderazgos surgidos en el grupo conllevan a la necesidad de generar un nuevo GI. Suele aparecer cuando los investigadores cuentan con la experiencia y formación para independizarse de aquel grupo que los formó, creando uno nuevo. Al respecto, Rey-
Rocha, Martín y Sebastián (2008), encontraron que 159 los integrantes que salen de un GI pueden generar nuevos grupos o vincularse a otros ya existentes, los cuales respondan a sus intereses y expectativas insatisfechos en el grupo inicial, fenómeno conocido como desegregación. Gil, Alcover, García y Rodríguez (2007) también plantean que los grupos son sistemas dinámicos que pueden modificarse o desaparecer a lo largo de un tiempo o diluirse y ramificarse, formando nuevos grupos o al contrario desapareciendo definitivamente.

Por último, el tercer escenario de transformación es cuando una experiencia previa del trabajo en grupo ha sido tan difícil, que no se encuentra una motivación significativa para continuar, o cuando los objetivos iniciales del grupo se cumplieron y no hay una nueva expectativa para justificar su permanencia. En este punto se da el fin del ciclo de vida del GI con la terminación y disolución del mismo, esta situación se puede deber a que no se dio un manejo óptimo de las crisis que se presentaron en la etapa anterior. Al respecto Schütz (1967, citado en Sánchez, 2002) afirma que se puede predecir una disolución o ruptura definitiva de un grupo, cuando sea incapaz de superar una fase determinada.

Es así como en la Figura 1 se esquematiza un modelo en espiral, para describir las fases del ciclo de vida de un GI (en este caso Psicología y Salud), bajo el supuesto de que las etapas se podrán vivir una y otra vez, con niveles crecientes de aprendizaje y complejidad, pues el grupo es un sistema vivo, que requiere de acomodaciones y adaptaciones constantes, ante las variaciones del ambiente interno y externo en el que se encuentre, postura que difiere de modelos lineales que se plantearon con anterioridad (Rey-Rocha, Martín y Sebastián, 2008; Hamui, 2011). 
Figura 1. Fases del ciclo de vida de un GI

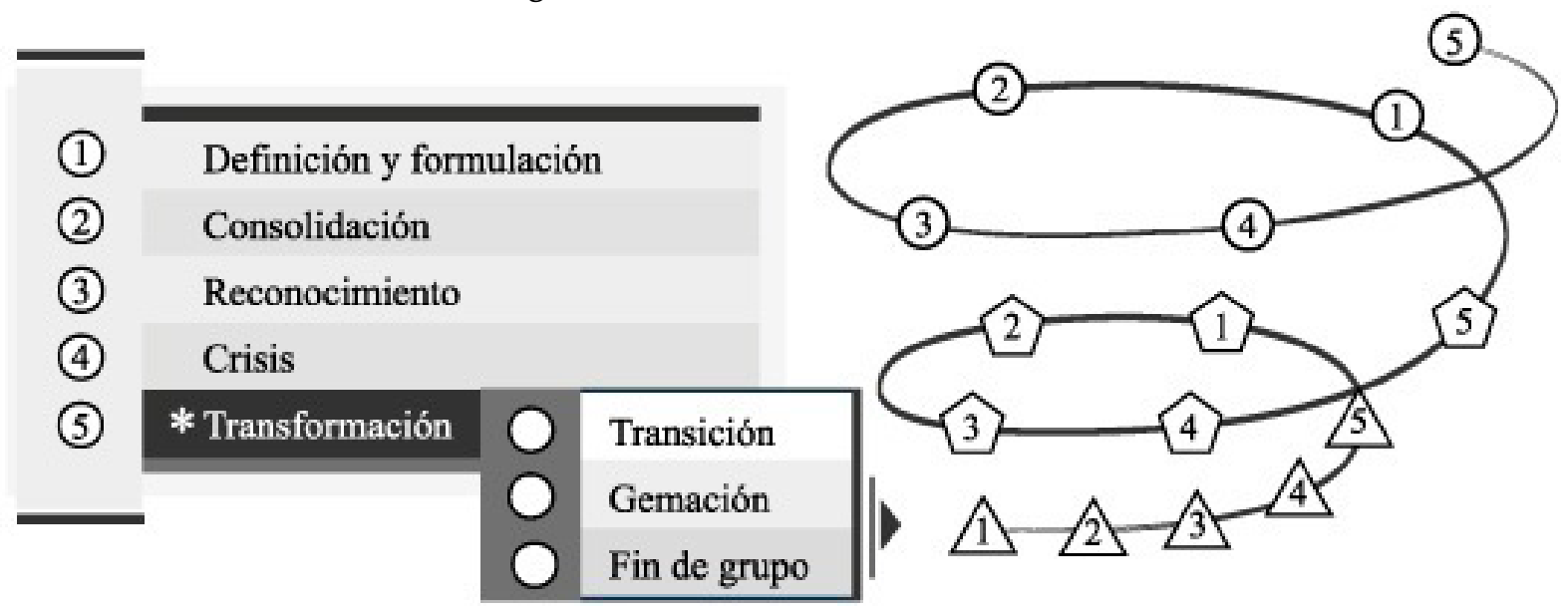

${ }^{*}$ En esta etapa se puede dar cualquiera de los 3 procesos

Fuente: elaboración de los autores.

\section{Aportes a la definición de grupo de investigación}

A partir de lo anterior, y como categoria central de análisis de este estudio, se ve la necesidad de ampliar la mirada que normalmente se tiene de un GI, de tal manera que se pueda comprender desde sus aspectos sociales e individuales, y no solo desde su productividad. Esta propuesta nace del análisis de las anteriores categorías sumado a la descripción de las vivencias manifestadas por los participantes. Es así como se proponen los siguientes elementos definitorios de un GI, entendiéndolo como un sistema vivo, el cual corresponde a un espacio social y de formación integral, en donde un conjunto de personas interactúan con el fin de aprender, investigar y generar nuevo conocimiento científico, a través del apoyo y gestión de procesos que respaldan su accionar como grupo. A continuación se profundizará cada elemento propuesto.

Grupo como sistema vivo, que requiere de adaptaciones constantes ante las variaciones del ambiente interno y externo en el que se encuentra, $y$ responde a determinadas etapas en el transcurrir de su ciclo de vida, «entonces creo que en ese ejercicio de autoevaluación, se dio la posibilidad de ver que muchas de las cosas no se estaban haciendo bien y entonces por eso se abrieron nuevas posibilidades» (DG).

Grupo como espacio social, es decir, un escenario en el que las personas se encuentran para sentirse «parte de» y construyen juntas desde su diversidad, lo cual implica un compromiso por la consolidación de un ambiente de trabajo saludable y enriquecedor, que garantice la validez y pertinencia social de la ciencia, tecnología e innovación: «Poder compartir periódicamente (...) con colegas y con estudiantes» (E-S1).

Grupo como espacio de formación integral, en el que el aprendizaje de los conocimientos, actitudes, hábitos y destrezas frente a la investigación, sea una oportunidad constante y necesaria, además de representar una ocasión de desarrollo integral, al comprender que antes que investigadores, el grupo se conforma de personas, con fortalezas y limitaciones, como lo manifiesta un integrante: «Son muchos los motivos que influyeron en mi decisión de hacer parte del grupo, sin embargo rescato como principal razón, las oportunidades académicas, el aprendizaje y el crecimiento personal como profesional que ofrecía el grupo Psicología y Salud» (E-S11). 
Grupo como estrategia de generación de conocimiento, dedicado a la gestión del conocimiento científico, tecnológico y de innovación, donde se realizan actividades guiadas hacia la construcción de nuevo conocimiento, las cuales se evidencian en la producción y publicación académica del grupo: «Yo creo que el grupo está a otro nivel y tiene un compromiso con la disciplina y con la región, y eso también obliga a que uno no puede quedarse quieto, tiene que estar generando conocimiento científico o conocimiento aplicado» (DG).

Grupo como referente de apoyo, que brinda el respaldo frente a las propuestas, proyectos e iniciativas de sus integrantes respondiendo a sus motivos personales, como base para la consolidación de un proyecto grupal: «(Las principales fortalezas del grupo han sido) la calidad del trabajo de sus integrantes, la autonomía que se otorga a los investigadores y el apoyo que se les brinda» (E-S4).

Y finalmente, grupo como un espacio de gestión de procesos, al propender por el desarrollo del talento humano, la organización, el funcionamiento administrativo y el aseguramiento de los recursos financieros necesarios para el adecuado cumplimiento de su misión, como por ejemplo, «es un grupo que apoya a sus integrantes en el desarrollo de proyectos, la participación en convocatorias y la difusión de resultados» (ES-4).

Estos elementos de definición encuentran una relación cercana a lo planteado por Bianco y Sutz (2014) al identificar que los aspectos importantes de un GI son: la interacción social, las expectativas de los integrantes, la estructura organizacional, la producción de saberes y, por último, el componente emocional, el cual facilita la conformación de la identidad de los integrantes del grupo.

Como limitaciones de este estudio, se evidenció que las publicaciones referidas a la dinámica social de un GI son escasas en el medio científico, especialmente en procesos de motivación de los investigadores y en modelos de ciclos de vida específicos de los GI. Debido a lo anterior, no se incluyen referencias de trabajos realizados en años recientes, sin embargo, se hizo uso de teoría clasica de grupos y de psicología de la motivación para poder comprender integralmente el funcionamiento individual y social del grupo Psicología y Salud. Se sugiere entonces, realizar más investigaciones frente al tema, que puedan profundizar y formular teoría con respecto a esta modalidad de grupos.

En el presente artículo se propuso un modelo propio de ciclo de vida de un GI, en este caso el grupo Psicología y Salud, que responde a su propia dinámica y el cual puede tener similitudes con otros grupos, sin embargo, no es algo que necesariamente todos los grupos vivan identicamente, puesto que el paso de una etapa a otra puede estar determinado por diferentes factores como la dinámica del grupo, las características de sus miembros, su trayectoria, la naturaleza de la tarea, las exigencias y contribuciones del entorno, entre otros. Por tanto, es necesario que se realicen investigaciones que nutran el modelo propuesto, permitan evaluar su aporte a una mayor comprensión del funcionamiento de los GI y faciliten su consolidación como un modelo robusto que responda a las particularidades de los diferentes grupos de investigación, en áreas diferentes a la psicología y en otro tipo de acciones científicas (investigación básica, desarrollo tecnológico, innovación, entre muchas otras).

\section{CONCLUSIONES}

El presente trabajo tenía como objetivos formular una propuesta teórica sobre el ciclo de vida de un GI, identificar los aspectos motivaciones de sus integrantes $y$, desde lo anterior, formular una nueva concepción de GI; aspectos que puedan aportar a una mayor comprensión de su dinámica interna y externa, y que permitirían brindar herramientas para reconocerlos no solo como unidades productivas de conocimiento, sino también, como sistemas vivos que necesitan políticas que apoyen su actividad científica, social y personal. De acuerdo con los resultados obtenidos, el primer aporte se 
centra en la importancia de identificar la motivación de cada integrante para pertenecer y permanencer en el GI. De esta forma, es fundamental que los objetivos del GI estén alineados a los intereses que tiene cada uno de los investigadores, y que, de esa manera, se promueva tanto la satisfacción como la permanencia de los mismos. Por ende, el reto para el grupo sería responder adecuadamente a dichas necesidades, con miras a mantener la motivación y compromiso de sus integrantes.

El modelo planteado de ciclo de vida para el GI Psicología y Salud, permite una mayor comprensión de cómo ha sido su funcionamiento y el afrontamiento de las situaciones presentes en cada etapa. En esa medida, cabe resaltar que el modelo se entiende desde una espiral ascendente, es decir, que en las nuevas etapas que vivirá el GI se demostrará el aprendizaje organizacional (tanto grupal como individual), poniendo de manifiesto que el modelo busca representar el fenómeno desde un interés descriptivo y no determinista.

Adicionalmente, se propone entender a los GI como sistemas dinámicos y complejos con funcionamientos diversos, los cuales orientan su labor a construir y difundir saberes científicos, resaltando la importancia de la motivación de los integrantes, las etapas de desarrollo que viven y los procesos de dinámica grupal que se pueden presentar, los cuales no deben ser dejados de lado, sino al contrario, deben articularse para que se pueda dar una aplicabilidad adecuada, propiciando la productividad, el bienestar de los integrantes y el crecimiento constante.

En conclusión, lo expuesto anteriormente nos permite comprender que un GI es mucho más que una unidad de producción de artículos, capítulos de investigación, patentes, ponencias o proyectos, por el contrario, más que máquinas, los investigadores deben ser reconocidos inicialmente desde su dimensión humana, colmada de intereses, inquietudes, expectativas y temores, los cuales ponen al servicio de la generación de nuevo conocimiento científico. Está en las instituciones y en los mismos grupos, apropiar estos elementos a la hora de formular políticas y estrategias destinadas a la gestión del conocimiento.

\section{REFERENCIAS}

Altopiedi, M., Hernández, E., \& López, J. (2015). Características relevantes de grupos de investigación destacados en Andalucía. Revista Iberoamericana de Educación Superior, 6(16), 126142. doi.org/10.1016/j.rides.2015.07.002

Bianco, M. \& Sutz, J. (2005). Las formas colectivas de la investigación universitaria. Revista Iberoamericana de Ciencia, Tecnología y Sociedad, 2(6), 25-44. Recuperado de http:/ / www.scielo. org.ar/pdf/cts/v2n6/v2n6a02.pdf

Bianco, M. \& Sutz, J. (2014). Veinte años de políticas de investigación en la Universidad de la República: aciertos, dudas y aprendizajes. Montevideo: Universidad de la República.

Blanco, A., Caballero, A., \& De la Corte, L. (2005). Psicología de los grupos. Madrid: Pearson Educación.

Ceballos, H., Garza, S. \& Cantu, F. (2018). Factors influencing the formation of intra-institutional formal research groups: group prediction from collaboration, organisational, and topical networks. Scientometrics, 114(1), 181-216. doi: 10.1007/s11192-017-2561-1

Clavijo, M. (2014). Introspección desde la formación para la investigación y la investigación formativa. Praxis \& Saber, 5(10), 93-119. doi. org/10.19053/22160159.3024

Colciencias (2017). Modelo de medición de grupos de investigación, desarrollo tecnológico o de innovación y de reconocimiento a investigadores del sistema nacional de ciencia, tecnología e innovación, año 2017. Bogotá, Colombia. Recuperado de http:// www.colciencias.gov.co/sites / default/files / 
upload / convocatoria / anexo_1._documento_ conceptual_modelo_medicion_de_grupos_e_ investigadores_2017_-_12_05_2017_protected. pdf

Colciencias (2017). Publicación de resultados finales de la convocatoria 781 de 2017. Bogotá, Colombia. Recuperado de http://www. colciencias.gov.co/sites / default/ files/ listado_ resultados_finales_-_781_de_2017_-_grupos_-_ firmados.pdf

Conde, Y., \& Castañeda, D. (2014). Indicadores de aprendizaje organizacional en grupos de investigación universitaria. Diversitas, 10 (1), 45-56. Recuperado de: http://revistas. usta.edu.co/index.php /diversitas/article/ view / 1564/1939

Crano, W., \& Brewer, M. (1973). Principles of research in social psychology. EE.UU.: McGrawHill.

Flick, U. (2004). Introducción a la investigación cualitativa. Madrid: Ediciones Morata/ Fundación Paideia Galiza.

Fuentes, C. (2015). Investigación en educación: de la práctica docente a los aspectos epistemológicos, éticos y sociales. Praxis \& Saber, 6(11), 235-244. doi.org/10.19053/22160159.3581

Gaete, R. (2010). La responsabilidad social universitaria como desafío para la gestión estratégica de la Educación Superior: el caso de España. Revista de Educación, 355, 109-133. Recuperado de http:/ / www.revistaeducacion. educacion.es / re355 / re355_05.pdf

Gil, F., Alcover, C., García, M., \& Rodríguez, F. (2007). Prácticas de psicología de los grupos, experiencias. Madrid: Pirámide.

Gómez, M., \& Montserrat, A. (2015). Factores influyentes de la gestión del conocimiento en el contexto de la investigación universitaria. Información, cultura y sociedad, 33, 29-46. Recuperado de http://www.redalyc.org/ articulo.oa?id=263042678003
Gómez, Y., \& Villalobos, F. (2014). Competencias para

la formulación de un proyecto de investigación. Guía metodológica del proyecto INVESTIC para docentes investigadores. San Juan de Pasto: Editorial Universidad de Nariño. Recuperado de http:/ / psicologiaysalud.udenar.edu.co/wp-content/ uploads / 2016 / 02 / Competencias-para-laformulacion-de-un-proyecto-de-investigacion. pdf

González, P. (1979). Motivación y productividad en la investigación científica española. Revista Española de Investigaciones Sociológicas, 7, 63-75. doi: $10.2307 / 40182761$

Hamui, M. (2011). Estructura organizativa y trayectoria de un grupo de investigación científica de relaciones internacionales. Perfiles educativos, 33(133), 51-67. Recuperado dehttp:/ / www.redalyc.org/articulo.oa?id=13219088004

Hernández, R., Fernandez, C., \& Baptista, P. (2014). Metodología de la investigación ( $6^{\circ}$ edición). México: McGrawHill.

Isohanni, M., Isohanni, I., \& Veijola, J. (2002). How should a scientific team be effectively formed and managed. Nordic Journal of Psychiatry, 56(2), 157-162. doi:10.1080/080394802753617999

Kuhn, T. (2006). La estructura de la revolución científica ( $3^{a}$ ed.). México, D. F.: Fondo de Cultura Económica.

Kyvik, S. (2013). The academic researcher role: enhancing expectations and improved performance. Higher Education, 65, 525- 538. doi: 10.1007/s10734-012-9561-0

Lastra, K. (2017). Investigación educativa en Argentina: impacto de las políticas de ciencia y tecnología en dos agencias del Estado, ANPCyT y CONICET. Revista Iberoamericana de Educación Superior, 8(21), 94-108. Recuperado de https: / / ries.universia.net/article/ view / 1128/ investigacion-educativa-argentina-impactopoliticas-ciencia-tecnologia-agencias-estadoanpcyt-conicet 
Londoño, F. (2005). Un análisis sobre la dinámica de los grupos de investigación en Colombia. De su conformación a su supervivencia. Investigación y desarrollo, 13(1),184-203.http: / / www.redalyc.org/pdf/268/26813108.pdf

Martínez, A. (2013). Nuevas posibilidades y retos para la investigación y el conocimiento en universidades. Diversitas, 9(1), 97-107. Recuperado de http://www.scielo.org.co/ pdf/dpp/v9n1/v9n1a08.pdf

Martínez, D. (2015). Participación social científico-tecnológica en la formación para la investigación de pregrado. TRILOGÍA. Ciencia, Tecnología y Sociedad, 7(12), 77-93. Recuperado de http://itmojs.itm.edu.co/ index.php / trilogia / article / view / 779 / pdf_21

McClelland, D. C. (1989). Motivational factors in health and disease. American Psychologist, 44, 675-683. doi.apa.org/journals/amp/44/4/675.pdf

Nieto, D., \& Gómez, N. (2016). Significado psicológico del concepto investigación en investigadores. Diversitas, 12(1), 109-121. Recuperado de http://www.scielo.org.co/ pdf/dpp/v12n1/v12n1a09.pdf

Omar, Z. \& Ahmad, A. (2014). Factor contributing to research team effectiveness: Testing a model of team effectiviness in an academic setting. International Journal of Higher Education, 3(3), 10-26. https://doi.org/10.5430/ijhe.v3n3p10

Ponce, H. (2007). La matriz FODA: alternativa de diagnóstico y determinación de estrategias de intervención en diversas organizaciones. Enseñanza e Investigación en Psicología, 12(1), 113-130. Recuperado de http: / / www.redalyc. org/pdf/292/29212108.pdf
Raymond,E.(2005).Lateorización anclada (grounded theory) como método de investigación en ciencias sociales: en la encrucijada de dos paradigmas. Cinta de Moebio, 23, 217-227. Recuperado de http: / / www.revistas.uchile.cl/index.php / CDM/article/viewFile/26082/27387

Rey-Rocha, J., Garzón, B. \& Martín, M. (2006). Scientists' Performance and Consolidation of Research Teams in Biology and Biomedicine at the Spanish Council for Scientific Research. Scientometrics, 69(2), 183-212. doi: 10.1007/s11192006-0149-2

Rey-Rocha, J., Martín, M., \& Sebastián, J. (2008). Estructura y dinámica de los grupos de investigación. ARBOR, Ciencia, Pensamiento y Cultura, 184, 743-757. doi:10.3989/arbor.2008. $i 732.219$

Romero, O. (1999). Crecimiento psicológico y motivaciones sociales. Mérida: ROGYA.

Sánchez, J. (2002). Psicología de los grupos. Teorías, procesos y aplicaciones. Madrid: McGrawHill.

Shaw, M., Robbin, R., \& Belser, J. (1981). Group dynamics: the psychology of small group behavior. United States of America: McGrawHill.

Strauss, A., \& Corbin, J. (2002). Bases de la investigación cualitativa. Técnicas y procedimientos para desarrollar la teoría fundamentada. Bogotá: Editorial Universidad de Antioquia.

Tuckman, B. (1965). Developmental sequence in small groups. Psychological Bulletin, 63(6), 384399. doi.org/10.1037/h0022100

Tuckman, B. W., \& Jensen, M. A. (1977). Stages in small group development revisited. Group and Organisation Studies, 2, 419-427. doi: 10.1177/105960117700200404 